\title{
Hepatic steatosis and steatohepatitis: a functional meta-analysis of sex-based differences in transcriptomic studies
}

José F. Català-Senent ${ }^{1,2}$ D , Marta R. Hidalgo ${ }^{1 *}$ (D), Marina Berenguer ${ }^{3,4,5,6}$ (D), Gopanandan Parthasarathy ${ }^{7}$ (D),

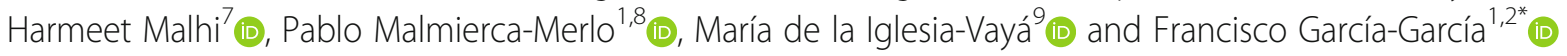

\begin{abstract}
Background: Previous studies have described sex-based differences in the epidemiological and clinical patterns of non-alcoholic fatty liver disease (NAFLD); however, we understand relatively little regarding the underlying molecular mechanisms. Herein, we present the first systematic review and meta-analysis of NAFLD transcriptomic studies to identify sex-based differences in the molecular mechanisms involved during the steatosis (NAFL) and steatohepatitis (NASH) stages of the disease.
\end{abstract}

Methods: Transcriptomic studies in the Gene Expression Omnibus database were systematically reviewed following the PRISMA statement guidelines. For each study, NAFL and NASH in premenopausal women and men were compared using a dual strategy: gene-set analysis and pathway activity analysis. Finally, the functional results of all studies were integrated into a meta-analysis.

Results: We reviewed a total of 114 abstracts and analyzed seven studies that included 323 eligible patients. The meta-analyses identified significantly altered molecular mechanisms between premenopausal women and men, including the overrepresentation of genes associated with DNA regulation, vinculin binding, interleukin-2 responses, negative regulation of neuronal death, and the transport of ions and cations in premenopausal women. In men, we discovered the overrepresentation of genes associated with the negative regulation of interleukin- 6 and the establishment of planar polarity involved in neural tube closure.

Conclusions: Our meta-analysis of transcriptomic data provides a powerful approach to identify sex-based differences in NAFLD. We detected differences in relevant biological functions and molecular terms between premenopausal women and men. Differences in immune responsiveness between men and premenopausal women with NAFLD suggest that women possess a more immune tolerant milieu, while men display an impaired liver regenerative response.

Keywords: Non-alcoholic fatty liver disease, Sex characteristics, Precision medicine, Computational biology, Transcriptome profiling

* Correspondence: mhidalgo@cipf.es; fgarcia@cipf.es

${ }^{1}$ Bioinformatics and Biostatistics Unit, Principe Felipe Research Center, Valencia, Spain

Full list of author information is available at the end of the article

C C The Author(s). 2021 Open Access This article is licensed under a Creative Commons Attribution 4.0 International License, which permits use, sharing, adaptation, distribution and reproduction in any medium or format, as long as you give appropriate credit to the original author(s) and the source, provide a link to the Creative Commons licence, and indicate if changes were made. The images or other third party material in this article are included in the article's Creative Commons licence, unless indicated otherwise in a credit line to the material. If material is not included in the article's Creative Commons licence and your intended use is not permitted by statutory regulation or exceeds the permitted use, you will need to obtain permission directly from the copyright holder. To view a copy of this licence, visit http://creativecommons.org/licenses/by/4.0/ The Creative Commons Public Domain Dedication waiver (http://creativecommons.org/publicdomain/zero/1.0/) applies to the data made available in this article, unless otherwise stated in a credit line to the data. 


\section{Background}

Non-alcoholic fatty liver disease (NAFLD) encompasses a spectrum of liver disorders-ranging from fat accumulation in hepatocytes (NAFL) to non-alcoholic steatohepatitis (NASH) - which can lead to the development of cirrhosis or liver cancer. While steatosis characterizes NAFL, NASH exhibits the additional histological features of inflammation, ballooning, and fibrosis. Awareness of NAFLD has recently increased due to its worldwide impact on health; indeed, NAFLD now represents the most common liver disease in developed countries [1]. While the estimated worldwide prevalence of NAFLD is around 25\%, it is more common in South America and the Middle East and less prevalent in Africa [2].

Of note, there exists significant interindividual heterogeneity regarding NAFLD progression, which has a considerable impact on the clinical consequences to the individual. Patients in the initial stage of the disease (NAFL) display a low risk of adverse outcomes [3]; however, progression to NASH increases the possibility of both hepatic and extrahepatic complications.

Interestingly, NAFLD has a higher prevalence in men and postmenopausal women than premenopausal women [4]. Indeed, the liver shows the second-largest amount of sexual dimorphism in humans [5], with both physiological and pathological hepatic processes, such as the detoxifying metabolism of cholesterol and the prevalence of hepatic diseases, respectively, differing between men and women [6]. Therefore, patient sex will likely affect NAFLD progression and treatment outcomes [7]; however, most NAFLD studies do not consider differences between sexes in their analysis [8]. We sought to explore the molecular mechanisms underpinning the likely existence of sex-related differences in NAFLD patients for the above-mentioned reasons.

In this context, we carried out the first (to the best of our knowledge) systematic review and meta-analysis of transcriptomic studies in NAFLD. We selected studies that had deposited data in the Gene Expression Omnibus (GEO) datasets database [9] to guarantee the eligibility of selected studies. We used these analyses to clarify the molecular mechanisms underpinning differences in NAFL and NASH in premenopausal women and men (postmenopausal women were not included in the analysis due to the overall low number of cases). Overall, sex-based differences may have important clinical implications for patients, and this knowledge may help to develop personalized approaches to NAFLD management.

\section{Materials and methods}

\section{Literature review}

A review was conducted in June 2020 following the Preferred Reporting Items for Systematic Reviews and
Meta-Analyses (PRISMA) statement guidelines [10]. The GEO datasets database was searched [9] using the keywords "NAFLD", "NAFL", "steatosis", "NASH", and "steatohepatitis" for transcriptomic studies published in English.

\section{Study exclusion criteria}

The following exclusion criteria were applied: (i) studies conducted in organisms other than humans, (ii) studies without information regarding the sex of participants or that did not include both sexes, (iii) studies without individuals from the NAFL and NASH stages of the disease, and (iv) studies in which the disease had not been diagnosed with a biopsy. In the latter case, the requirement for NAFL and NASH diagnoses by biopsy was added as less invasive methods (such as conventional imaging techniques) cannot accurately detect mild steatosis or differentiate NAFL from NASH [11]. This step aimed to minimize the input of false-positive data into the statistical analyses caused by an incorrect disease stage classification.

\section{Bioinformatics analysis}

The same three-step transcriptomic analysis strategy was applied to each separate study: (i) data acquisition and preprocessing, (ii) differential gene expression and functional enrichment analysis, and (iii) differential pathway activation and functional analysis. The functional results of all studies were integrated using meta-analytical techniques (Fig. 1). Bioinformatics analysis was carried out using the programming language $R$ 3.6.0 [12]; information regarding the packages used and their version numbers are provided in the supplementary material.

\section{Data acquisition and preprocessing}

During data preprocessing, the nomenclature of the distinct disease stages (by grouping patients into Control, NAFL, and NASH groups) and the association of the probe identifiers with their corresponding genes were standardized. In those studies containing treated and untreated patients, treated patients were eliminated from further analysis. For studies that used microarrays, probe codes were transformed to their respective Entrez identifiers from the NCBI database. For repeated probes, the median of their expression values was calculated. When available, data normalized by the original authors of the studies were used. Otherwise (studies GSE61260 and GSE66676), raw data were normalized using the Robust Multichip Average (RMA) algorithm [13]. The gene nomenclature in RNA-Seq studies was also standardized to the Entrez identifiers, and the raw data matrix was processed using the TMM standardization method [14] followed by a log transformation of the data. 


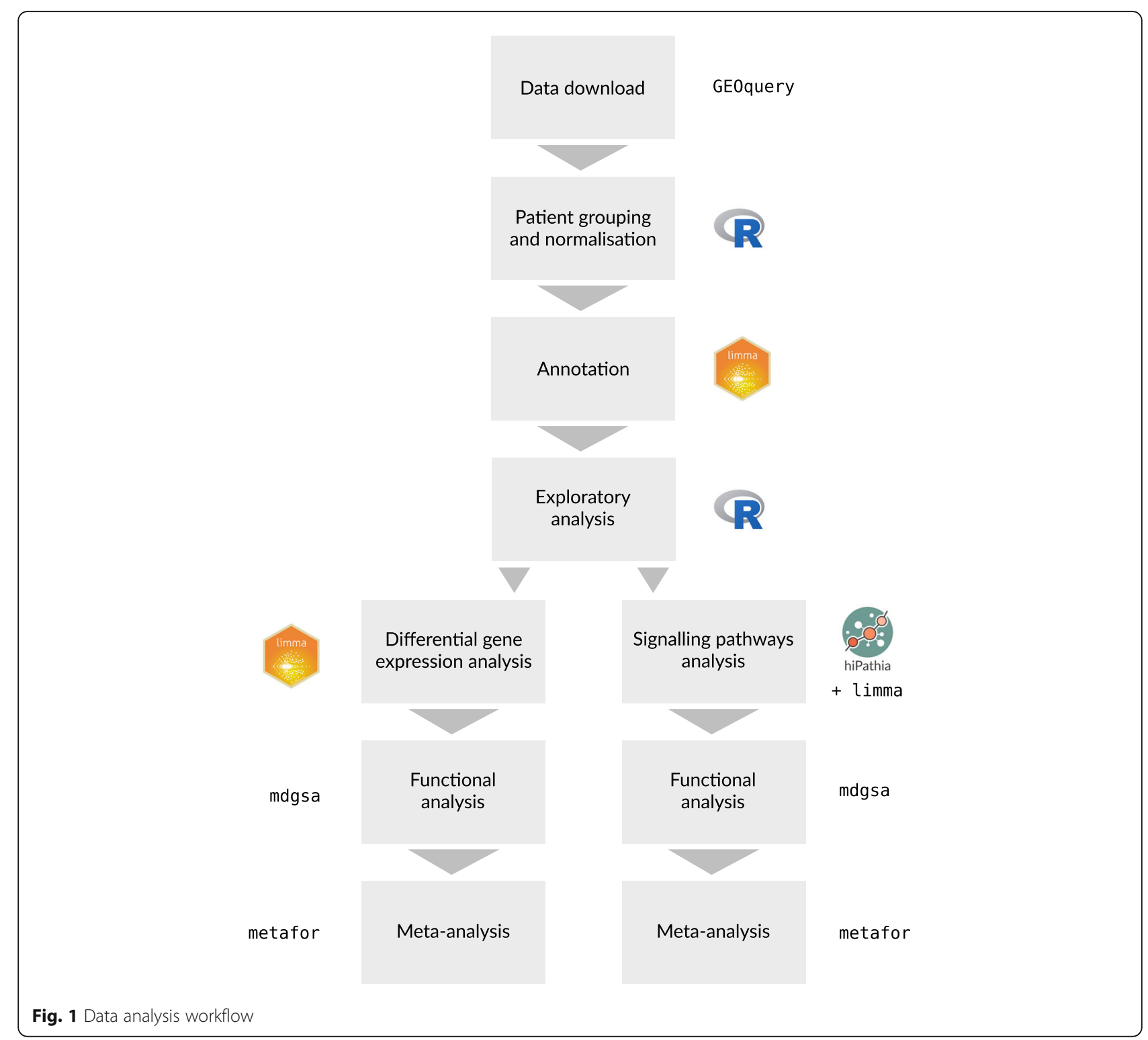

Women were separated into premenopausal and postmenopausal groups to meet the objective of this work. As selected studies failed to include this specific information, women aged under 50 years were assumed as premenopausal based on studies indicating an average age of menopause at around 48-52 years [15, 16].

After data normalization, the detection of possible anomalous effects within the studies was carried out by completing an exploratory clustering and a principal component analysis (PCA).

\section{Differential gene expression and functional enrichment analysis}

Differential expression analyses of selected studies were carried out using the limma and edgeR packages $[17,18]$ to detect differentially expressed genes when comparing
NAFL and NASH in premenopausal women versus men. A linear model was adjusted for each gene, which included possible batch effects, contrasting (NASH.W NAFL.W) and (NASH.M - NAFL.M), where NASH.W, NAFL.W, NASH.M, and NAFL.M corresponded to NASH-affected premenopausal women, NAFL-affected premenopausal women, NASH-affected men, and NAFL-affected men, respectively. Statistics regarding the differential expression were calculated and $p$ values adjusted using the Benjamini \& Hochberg (BH) method [19].

Based on the differential gene expression results, functional enrichment analysis was then performed using gene set analysis (GSA) [20]. First, genes were ordered according to their $p$ value and the sign of the contrast statistic. Next, GSA was performed using the logistic 
regression model implemented in the $m d g s a \mathrm{R}$ package [21] along with their corresponding functional annotations obtained from the Gene Ontology (GO) [22] and Kyoto Encyclopedia of Genes and Genomes (KEGG) PATHWAY [23] databases.

Due to their hierarchical structure, the gene annotations with GO terms applied in the mdgsa package were propagated to inherit the annotations of the ancestor terms. Excessively specific or generic annotations (blocks smaller than ten or larger than 500 words) were subsequently filtered out. Finally, functions with a $\mathrm{BH}-$ adjusted $p$ value under 0.05 were considered significant.

For the two functional elements (GO terms and KEGG paths), the number of overrepresented elements shared by the studies were analyzed. These results were graphically represented as UpSet plots [24] to demonstrate the common elements between the different sets. First, the overrepresented elements were compared as separate graphs to detect the common functions in each group: GO terms in premenopausal women, GO terms in men, KEGG pathways in premenopausal women, and KEGG pathways in men. Next, any significant GO terms in each study were visualized in the same plot to highlight significant terms with different signs among the studies.

\section{Differential pathway activation and functional analysis}

The hipathia algorithm [25] was used to perform Pathway Activity Analysis (or PAA). This method transforms gene expression values for stimulus-response signaling sub-pathways into activation levels that ultimately trigger cellular responses. In this current study, the hipathia $\mathrm{R}$ package was used to analyze 1654 GO terms and 142 UniProt functions [26] associated with 146 KEGG routes [23]. The activation signal of each sub-pathway was computed from gene expression values using this algorithm. These values were used to detect significant differential activations in the (NASH.W - NAFL.W) (NASH.M - NAFL.M) contrast pair (as defined above). The functional annotations included in hipathia and the differential activation results were integrated using the GSA method to identify differences at the functional level (GO terms and UniProt functions) in relation to NAFLD stages.

\section{Meta-analysis}

Once the gene functional analysis was applied to each study, a functional meta-analysis was carried out to summarize the results. Similarly, once the pathway functional analysis was applied to each study, the results were summarized via a homologous functional metaanalysis.

Both meta-analyses were performed following the methodology described in detail by García-García [27]. Briefly, the metafor $\mathrm{R}$ package [28] was used to evaluate the combined effect within a random-effects model, which more precisely detects overrepresented elements when compared with performing studies individually at higher statistical power. Likewise, variability in the individual studies was considered in the global estimation of the measured effect so that less-variable results had a higher weight in the overall calculation of the logarithm of the odds ratio (LOR) [29]. Incorporating the variability between experiments in the model of random effects provides more statistically robust results and better integration of selected studies. Finally, the suitability of this selection was confirmed by evaluating the heterogeneity of each study in the global model and the application of cross-validation techniques.

For each meta-analysis and function, $p$ values (adjusted using the $\mathrm{BH}$ method), LORs, and 95\% confidence intervals (CIs) were calculated. A term was considered significant when its adjusted $p$ value was lower than 0.05; a positive LOR in significant functions indicated a greater overrepresentation in premenopausal women than in men. In contrast, a negative LOR indicated higher representation in men than in premenopausal women. Finally, funnel plots and forest plots were used to assess the variability and measure each study's contribution to the meta-analysis.

A total of 8223 elements were analyzed in the gene functional meta-analysis (7994 GO terms and 229 KEGG pathways); 1654 GO terms and 142 UniProt functions [26] were assessed in the functional pathway metaanalysis in association with 146 KEGG pathways [23].

\section{Availability of data and materials}

The large volume of data and results generated in this study is freely available through the metafun-NAFLD platform: https://bioinfo.cipf.es/metafun-NAFLD. Access allows any user to analyze the results shown in this manuscript and review other results that may be of interest. The front-end was developed using the Bootstrap library. All graphics used in this tool were implemented with Plot.ly except for the exploratory analysis cluster plot, which was generated with the ggplot2 package.

In this study, transcriptomic data from the Gene Expression Omnibus database with accession numbers GSE48452, GSE61260, GSE66676, GSE83452, GSE89632, GSE126848, and GSE130970 were analyzed.

\section{Results}

\section{Systematic review and exploratory analysis}

As shown in the PRISMA flow diagram (Fig. 2), our systematic review yielded 114 non-duplicated studies. After applying the previously described exclusion criteria, we employed a total of seven studies in our overall analysis. We excluded studies for the following reasons: studies not in humans or unrelated to NAFLD $(n=55)$, in vitro 

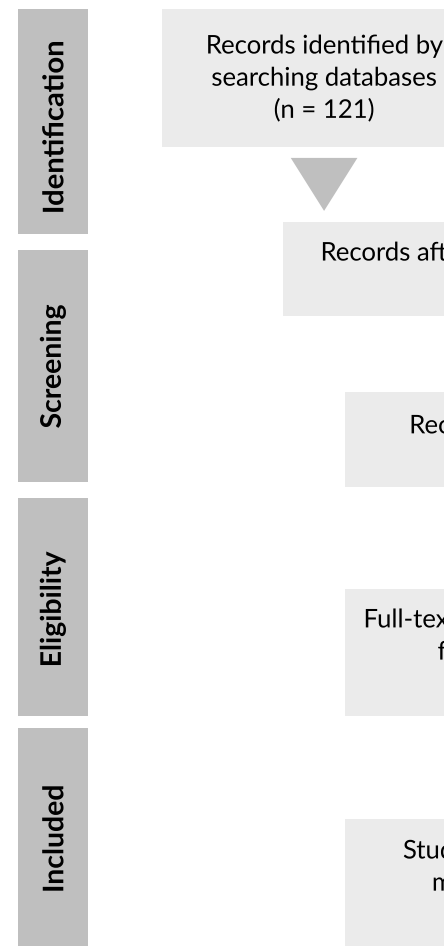

Records after duplicates removed $(n=114)$

\section{Additional records}

identified via other sources $(n=0)$

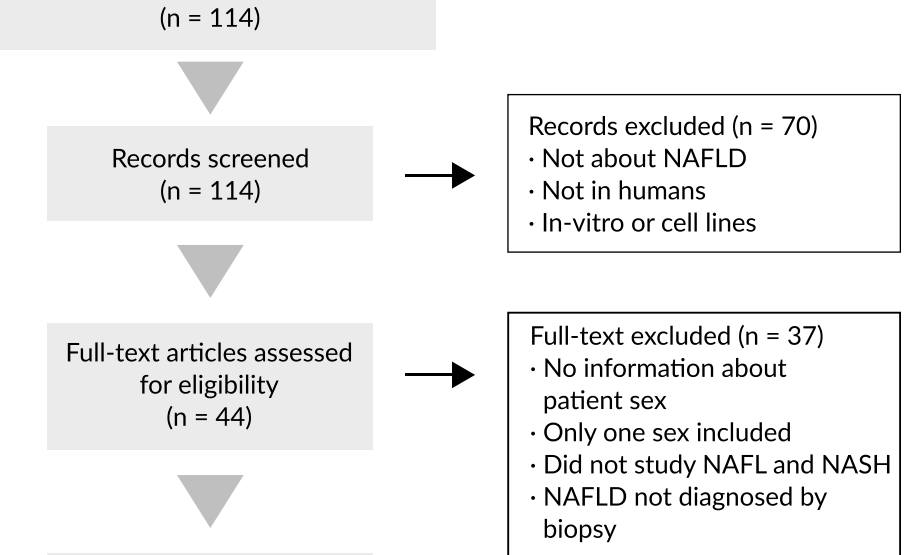

Studies included in meta-analysis $(n=7)$

Fig. 2 Flow diagram of our systematic review of the literature and selection of studies for meta-analysis-according to the PRISMA statement guidelines

studies $(n=15)$, lack of information regarding sex or inclusion of only one sex $(n=24)$, no patients in NAFL and NASH disease stages $(n=12)$, and diagnosis not based on histology $(n=1)$. The strict nature of these inclusion and exclusion criteria allowed the selection of comparable studies to ensure the reliability of the subsequent analytical strategies.

The seven selected studies [30-36] included a total of 323 eligible patients (Table 1); 164 individuals were men and 159 women under the age of 50 (49.2\% men and $50.8 \%$ women, respectively). By disease stage, 148 belonged to the NAFL group and 175 to the NASH group (45.8 and $54.2 \%$, respectively).

As previously indicated, we only used data from nontreated individuals in study GSE83452. Table 1 demonstrates the number of individuals from each study eligible for inclusion based on our aforementioned criteria. In an exploratory analysis, PCA of the GSE83452 study demonstrated that samples clustered into two groups. Further analysis of this study identified technical factors as the source of this grouping rather than any other clinical characteristic, as samples grouped according to their

Table 1 Characteristics of the studies selected for inclusion in our analysis in terms of disease stage, sex, BMI, and patient age

\begin{tabular}{|c|c|c|c|c|c|c|}
\hline \multirow[t]{3}{*}{ GEO accession, Authors, Year [Ref.] } & \multicolumn{4}{|c|}{ Patients by disease stage and sex } & \multirow{3}{*}{$\begin{array}{l}\text { Age } \\
\text { Mean + SD }\end{array}$} & \multirow{3}{*}{$\begin{array}{l}\text { BMI } \\
\text { Mean + SD }\end{array}$} \\
\hline & \multicolumn{2}{|c|}{ Premenopausal women } & \multicolumn{2}{|l|}{ Men } & & \\
\hline & NAFL & NASH & NAFL & NASH & & \\
\hline GSE48452 / Ahrens M. et al. 2013 [30] & 10 & 9 & 4 & 4 & $41.62 \pm 9.35$ & $47.18 \pm 11.34$ \\
\hline $\begin{array}{l}\text { GSE61260 / } \\
\text { Horvath S. et al. } 2014 \text { [31] }\end{array}$ & 10 & 7 & 12 & 12 & $41.51 \pm 9.09$ & $51.55 \pm 10.40$ \\
\hline GSE66676 / Xanthakos S.A. et al. 2015 [32] & 20 & 4 & 6 & 3 & $17.14 \pm 1.66$ & $53.55 \pm 8.86$ \\
\hline GSE83452 / Lefebvre P. et al. 2017 [33] & 32 & 35 & 6 & 46 & $39.61 \pm 11.51$ & $\begin{array}{l}\text { N/D } \\
\text { (> } 25 \text { or obese) }\end{array}$ \\
\hline GSE89632 / Arendt B.M. et al. 2015 [34] & 2 & 4 & 14 & 9 & $39.55 \pm 8.19$ & $30.21 \pm 5.51$ \\
\hline GSE126848 / Suppli M.P. et al. 2019 [35] & 6 & 4 & 9 & 12 & $40 \pm 13^{a}$ & N/D (> 30) \\
\hline GSE130970 / Hoang S.A. et al. 2019 [36] & 4 & 12 & 13 & 14 & $44.72 \pm 9.80$ & N/D \\
\hline
\end{tabular}

${ }^{a}$ The information corresponding to the patients in this study was kindly provided by the author of the study, although individual patient ages were not available 
order (the first 64 samples grouped into one cluster and the last 88 into another). We considered this a batch effect, which we accounted for in our subsequent analyses.

\section{Individual analysis}

The functional analysis of individual genes highlighted significant results overrepresented in both the groups of premenopausal women and men (summarized in Table 2). Analysis (using UpSet plots) of the common terms or pathways in the different studies demonstrated no common element in more than four studies (Supplementary Figure 1); in fact, we discovered opposite results for some elements in different studies (Fig. 3a). The functional analysis of individual pathways only exhibited significant results for the GSE83452 study (20 sub-paths and 96 GO terms).

\section{Meta-analysis}

We performed separate meta-analyses using the results of the two previous approaches to obtain a measure of the overall effect of each of the 8223 elements evaluated (GO terms, KEGG pathways, or UniProt functions) throughout all individual studies, i.e., the LOR and its confidence interval. This effect indicated the magnitude of the overexpression of each term (the LOR value) and its direction (the LOR sign). This sign also helped to identify the group in which the element is overrepresented. In our analysis, positive signs indicate overrepresentation in premenopausal women and negative signs as overrepresentation in men.

Along with the LOR, the meta-analysis also provides an adjusted $p$ value (FDR) that indicates the significance of the results. As a result of our meta-analysis, we identified thirteen significant GO terms (adjusted $p$ value < $0.05)$, which refer to the molecular mechanisms altered in our comparison (detailed in the following sections and Table 3). Figure 3b shows, for one function, the LOR of each study and the joint LOR of the metaanalysis. Supplementary Figures 2 and 3 detail the results of all significant elements. We confirmed the absence of bias and heterogeneity in all cases, as in Fig. 3c (Supplementary Figures 4 and 5).

\section{Gene functional meta-analysis}

The functional meta-analyses of the individual gene differential expression pipeline results provided a total of nine significant GO terms (Table 3 and Fig. 4) but no significant KEGG pathways. Among the significant GO terms, we identified six overrepresented in premenopausal women; these elements encompassed a broad spectrum of biological processes and molecular functions, including "RNA polymerase II regulatory region $D N A$ binding" (GO:0001012), "RNA polymerase II regulatory region sequence-specific DNA binding" (GO: 0000977), "Vinculin binding" (GO:0017166), "Cellular response to interleukin-2" (GO:0071352), "Negative regulation of neuron death" (GO:1901215), and "Response to interleukin-2" (GO:0070669). We identified three terms overrepresented in men: "Negative regulation of interleukin-6 secretion" (GO:1900165), "Establishment of planar polarity involved in neural tube closure" (GO:0090177), and "Regulation of establishment of planar polarity involved in neural tube closure" (GO:0090178).

\section{Pathway functional meta-analysis}

The functional meta-analysis of the individual results in the pathway differential activation pipeline returned four significant GO terms. These terms were overrepresented in premenopausal women compared with men, with a LOR value lower than 0.25 (Table 3 and Fig. 4). These terms relate to the transport of ions and cations: "Cation transmembrane transport" (GO:0098655), "Metal ion transport" (GO:0030001), "Regulation of cation transmembrane transport" (GO:1904062), and "Inorganic cation transmembrane transport" (GO:0098662). The metaanalysis of the UniProt functions failed to provide significant results.

Table 2 Number of significant GO terms and KEGG pathways in individual studies after gene functional analysis. Positive and negative logarithms of the odds ratio (LORs) demonstrate overrepresentation in premenopausal women and men, respectively

\begin{tabular}{|c|c|c|c|c|}
\hline & \multicolumn{2}{|l|}{ GO terms } & \multicolumn{2}{|c|}{ KEGG pathways } \\
\hline & $\begin{array}{l}\text { Positive LOR } \\
\text { (Pre. women) }\end{array}$ & $\begin{array}{l}\text { Negative LOR (Men) } \\
\text { (Men) }\end{array}$ & $\begin{array}{l}\text { Positive LOR } \\
\text { (Pre. women) }\end{array}$ & $\begin{array}{l}\text { Negative LOR } \\
\text { (Men) }\end{array}$ \\
\hline GSE48452 & 166 & 642 & 5 & 22 \\
\hline GSE61260 & 249 & 35 & 5 & 6 \\
\hline GSE66676 & 721 & 168 & 30 & 2 \\
\hline GSE83452 & 90 & 931 & 2 & 25 \\
\hline GSE89632 & 52 & 67 & 2 & 1 \\
\hline GSE126848 & 1168 & 150 & 55 & 5 \\
\hline GSE130970 & 1244 & 407 & 31 & 20 \\
\hline
\end{tabular}




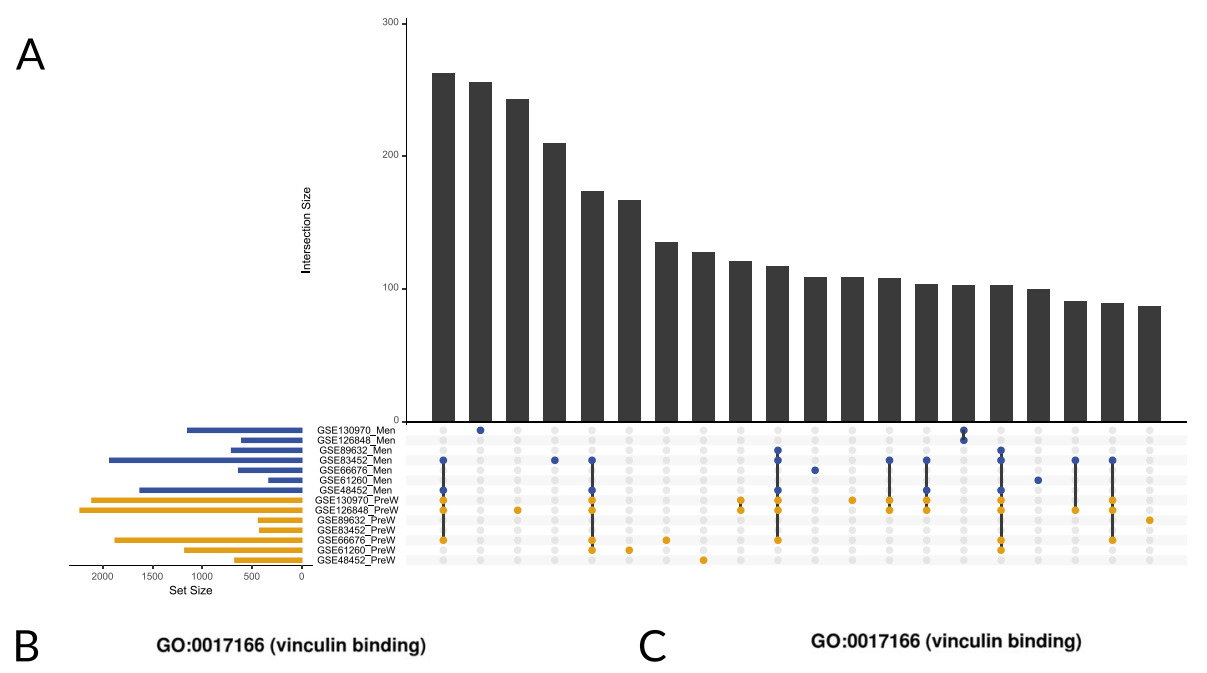

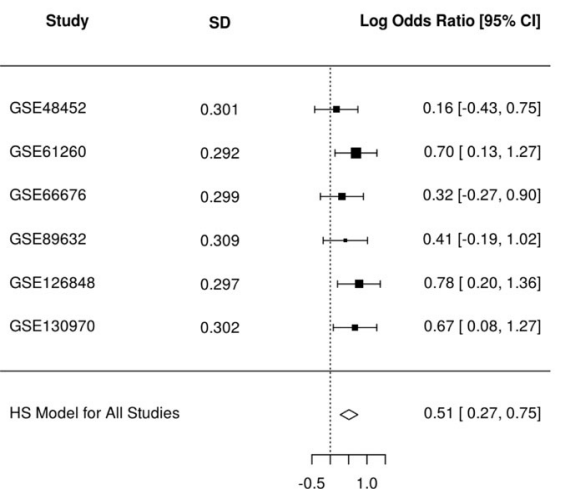

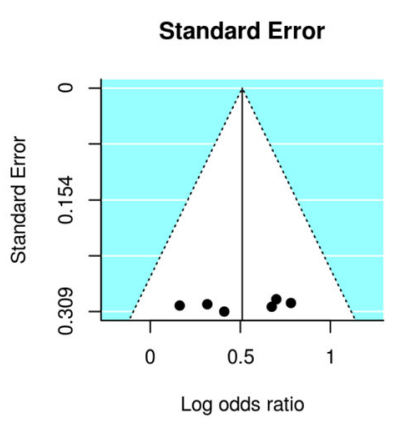

Fig. 3 Representation of results of the bioinformatics analysis. a UpSet plot showing the number of common results throughout individual studies in our functional gene analysis. Only the twenty most abundant interactions are shown. Horizontal bars indicate the number of significant elements in each study (premenopausal [orange] women and men [blue]). The vertical bars indicate the common elements in the sets, indicated with dots under each bar. The single points represent the number of unique elements in each group. Those vertical bars with dots of both colors beneath indicate significant amounts of molecular bases in men and women, in a contradictory way, in different studies. $\mathbf{b}$ Example of a forest plot from the meta-analysis. This representation allows us to observe the LOR and confidence interval of the GO terms in each study (horizontal bars) and the meta-analysis (diamond). c Example of a funnel plot from the meta-analysis. Dots in the white area indicate the absence of bias and heterogeneity

\section{Metafun-NAFLD platform}

To contribute to data sharing and support the development of subsequent related studies, we generated the Metafun-NAFLD platform (https://bioinfo.cipf.es/ metafun-NAFD). This web tool contains the detailed results of all the steps of the bioinformatics pipeline and allows simple interactive exploration by any interested party.

\section{Discussion}

Although previous studies have analyzed pathobiological mechanisms in NAFLD and (separately) sexual differences in metabolic regulation, the impact of sex on NAFLD pathobiology remains incompletely defined. Epidemiological studies have reported a higher prevalence of NAFLD in men and postmenopausal women than premenopausal women [4]. To better understand the molecular basis of sex-based differences, we present a systematic review and functional meta-analysis of publicly available genomic studies that analyze the differences in NAFLD progression between premenopausal women and men.

Despite the limitations of our approach (presence of studies with different sample sizes and types of platforms), meta-analysis techniques can integrate the selected studies. The individual results of the seven selected studies failed to identify any consistently different GO or KEGG terms between groups across all studies, a finding perhaps related to small sample sizes, underlying genetic and environmental variance in the source population, and methodological heterogeneity. Surprisingly, as shown in Fig. 3a, we found certain 
Table 3 Significant functions in the functional meta-analyses. The logarithm of the odds ratio (LOR), its confidence interval (LOR-CI), the adjusted $p$ value, and the group in which the function was overexpressed are shown ( $W$, women; $M$, men)

\begin{tabular}{|c|c|c|c|c|c|c|}
\hline & GO ID & Name & LOR & LOR-Cl & Adj. $p$ value & Group \\
\hline \multirow{9}{*}{$\begin{array}{l}\text { Gene functional } \\
\text { meta-analysis }\end{array}$} & GO:0001012 & RNA polymerase II regulatory region DNA binding & 0.132 & {$[0.079,0.185]$} & 0.008 & W \\
\hline & GO:1900165 & Negative regulation of interleukin- 6 secretion & -0.762 & {$[-1.088,-0.437]$} & 0.018 & M \\
\hline & GO:0000977 & $\begin{array}{l}\text { RNA polymerase II regulatory region sequence-specific } \\
\text { DNA binding }\end{array}$ & 0.131 & {$[0.073,0.188]$} & 0.02 & W \\
\hline & GO:0017166 & Vinculin binding & 0.511 & {$[0.271,0.75]$} & 0.035 & W \\
\hline & GO:0071352 & Cellular response to interleukin-2 & 0.467 & {$[0.248,0.687]$} & 0.035 & W \\
\hline & GO:0090177 & $\begin{array}{l}\text { Establishment of planar polarity involved in neural } \\
\text { tube closure }\end{array}$ & -0.514 & {$[-0.748,-0.279]$} & 0.035 & M \\
\hline & GO:0090178 & $\begin{array}{l}\text { Regulation of establishment of planar polarity involved } \\
\text { in neural tube closure }\end{array}$ & -0.523 & {$[-0.768,-0.279]$} & 0.035 & M \\
\hline & GO:1901215 & Negative regulation of neuron death & 0.116 & {$[0.06,0.171]$} & 0.043 & W \\
\hline & GO:0070669 & Response to interleukin-2 & 0.446 & {$[0.23,0.662]$} & 0.046 & W \\
\hline \multirow{4}{*}{$\begin{array}{l}\text { Pathway functional } \\
\text { meta-analysis }\end{array}$} & GO:0098655 & Cation transmembrane transport & 0.194 & {$[0.11,0.279]$} & 0.012 & W \\
\hline & GO:0030001 & Metal ion transport & 0.141 & {$[0.074,0.208]$} & 0.027 & W \\
\hline & GO:1904062 & Regulation of cation transmembrane transport & 0.216 & {$[0.119,0.32]$} & 0.027 & W \\
\hline & GO:0098662 & Inorganic cation transmembrane transport & 0.186 & {$[0.093,0.278]$} & 0.038 & W \\
\hline
\end{tabular}

functions overrepresented in both groups in different studies. A meta-analysis, such as that carried out in this study, can help to improve the identification of consistent, relevant differences across studies, subject to the caveat that patients in each study may have other differences in clinical characteristics. A meta-analysis also increases the overall sample size and hence the strength of findings, which is of particular relevance to diseases such as NAFLD that displays high levels of heterogeneity. Indeed, systematic reviews and meta-analyses represent widely employed strategies in studying sex-based differences in other diseases [37-39].

Our functional meta-analysis identified a total of thirteen GO terms (Table 3) that displayed differences between men and premenopausal women using two different approaches-differentially expressed genes (nine terms) and differentially activated pathways (four terms). These terms broadly related to processes such as inflammation, cell binding, and the establishment of polarity during neural tube closure.

A functional meta-analysis of differentially expressed genes identified differences in nine significant GO terms. Among these, our results established the overrepresentation of the "Vinculin binding" (GO:0017166) in premenopausal women compared with men. The vinculin cytoplasmic actin-binding protein is enriched in focal adhesions and adherens junctions to contribute to junction stability [40] and plays a vital role as a regulator of apoptosis [41]. Of note, although "cellular adhesion" represents a broad set of processes rather than a specific biologic pathway, this term was previously reported as a GO term consistently enriched in male NASH patients
[42]. Furthermore, Arendt et al. identified differentially expressed genes related to cell-cell adhesion (ANXA2 and $M A G$ ) between NAFL and NASH patients [34].

Our analysis also identified sex-based differences related to the inflammatory response, specifically the expression of cytokine-related terms. We found "Negative regulation of interleukin-6 secretion" (GO:1900165) overrepresented in men and "Cellular response to interleukin-2" (GO:0071352) and "Response to interleukin-2" (GO:0070669) overrepresented in premenopausal women. These terms relate to interleukin 2 (IL2) and interleukin 6 (IL-6), which displayed LOR values close to or above 0.5 , thereby indicating a substantial difference between the groups. IL-2 is a known regulator of $\mathrm{T}$ cell responses, with low IL-2 levels regulating $\mathrm{T}$ cell central tolerance via the formation of regulatory $\mathrm{T}$ cells [43]. Higher IL-2 levels induce T cell proliferation and differentiation into memory $\mathrm{T}$ cells. IL-2 receptors are also expressed by $\mathrm{B}$ cells and cells of the innate immune system [44]. Interestingly, IL-2 signaling is deficient in many chronic liver diseases [45], and levels of soluble IL-2 receptor alpha were found to be higher in children with NASH and advanced fibrosis [46]. Our results suggest that low-level IL-2-induced immune tolerance may mitigate the risk of developing NASH in premenopausal women and raise the intriguing possibility of restoring low-level IL-2 signaling in men as a potential treatment for NAFLD.

IL-6 has been previously implicated in NASH, with increased hepatic expression correlating with disease severity and insulin resistance [47]. IL-6 also regulates hepatic regeneration [48] and represents a significant driver of 


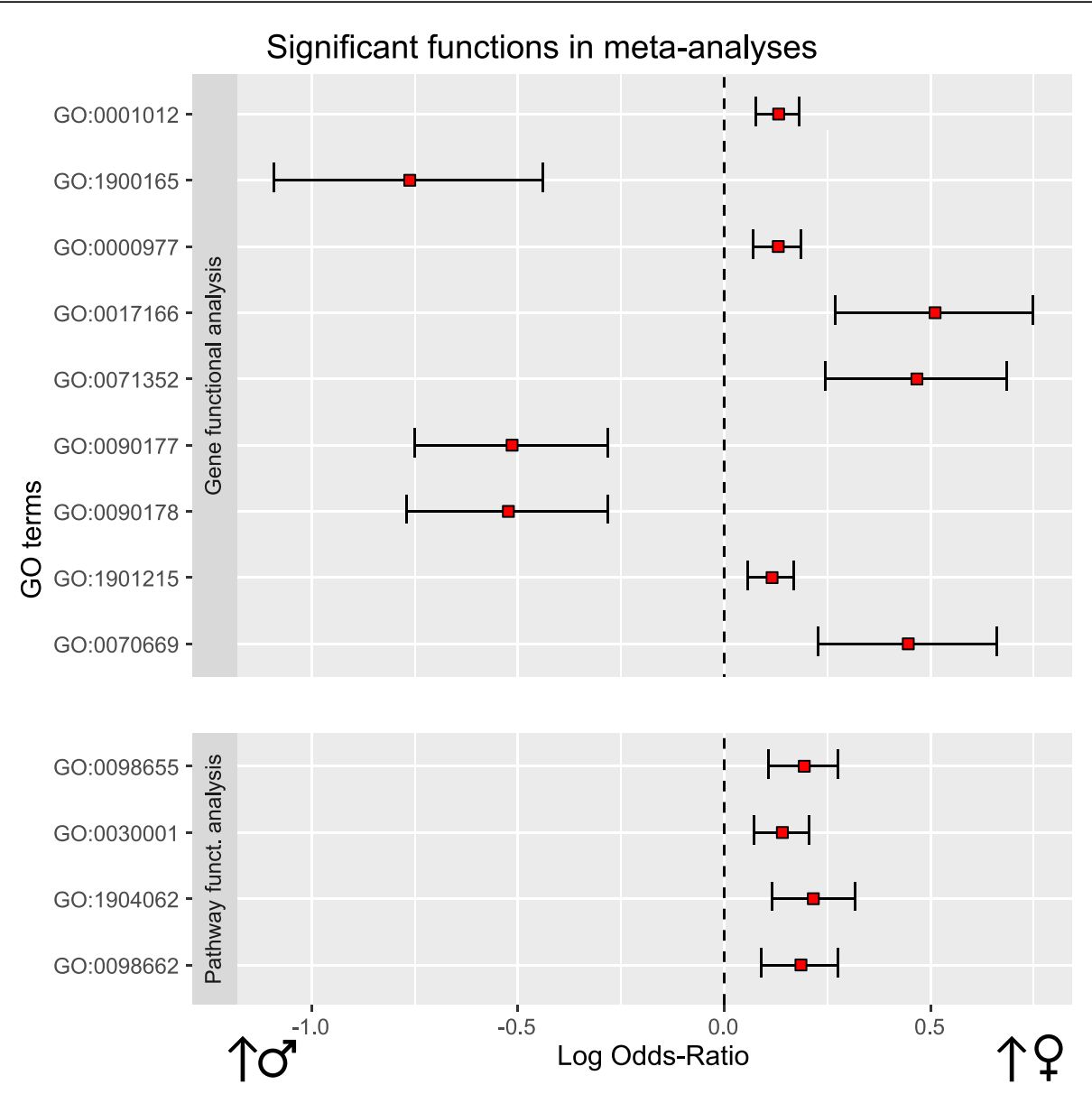

Fig. 4 Differential functional profiling by sex. This plot shows significant functional terms of each meta-analysis. On the right: biological functions over-represented in women. On the left: biological functions more overrepresented in men. For each function, the LOR (red square) and confidence interval have been represented

hepatic carcinogenesis [49]. In NAFLD, IL-6 may enhance hepatic repair and regeneration; however, IL-6 may also promote insulin resistance and hepatocyte apoptosis, thus contributing to NASH development $[48,50]$. Indeed IL-6 gene polymorphisms have been associated with increased susceptibility to chronic liver disease [51].

These results suggest that differences in immune mechanisms between men and women may represent one possible factor in the sex-differences observed in NAFLD. For example, the macrophage phenotype in male NAFLD mice presents as pro-inflammatory and pro-fibrotic, but pro-resolution and anti-fibrotic in female mice [52]. We hypothesize that women may suffer from a decreased immune response to lipotoxicity, whereas men may suffer from an impaired liver-healing response to chronic injury perhaps related to deficient IL-6 signaling. These ideas require further examination and validation in additional studies.

A recent study by Herrera-Marcos et al. detected sexbased differences in the control of the Cidec/Fsp27 $\beta$ gene in the liver, finding that gene expression correlated with the presence and density of liver lipid droplets in mice fed a high-fat diet [53]. Matsusue suggested a role for Cidec/Fsp27 $\beta$ as a neuronal activator in the steatotic liver [54]; therefore, the significant association with the function "Negative regulation of neuron death" (GO: 1901215) could relate to the differences detected in this previous study. Upon restricting the gene ontology analysis to Homo sapiens, we found the components of the "Negative regulation of neuron death" pathway (GO: 1901215) as broad regulators of apoptosis. This finding may suggest an increase in liver cell apoptosis in men who develop advanced NASH at a younger age than women, as premenopausal women possess a level of protection. The observation that hepatocyte apoptosis represents a prominent feature of progressive NASH agrees with this hypothesis [55].

Interestingly, we discovered other significant GO terms that had not previously been associated with NAFLD. For instance, we found the overrepresentation 
of "Establishment of planar polarity involved in neural tube closure" (GO:0090177) and "Regulation of establishment of planar polarity involved in neural tube closure" (GO:0090178) in men with a LOR exceeding 0.5 (-0.514 and -0.523). Restricting analyses to Homo sapiens demonstrated that these GO terms relate to the Wnt and frizzled family of proteins. Wnt ligands and their cognate receptor frizzled represent critical determinants of liver development, zonation, regeneration, and responses to liver injury [56]. Their overrepresentation in male NASH patients could indicate the activation of disease repair pathways as a compensatory response to ongoing liver injury [57], as observed in preclinical models of NASH.

Our functional pathway meta-analysis identified four significant GO terms involved in cation transport: "Cation transmembrane transport" (GO:0098655), "Metal ion transport" (GO:0030001), "Regulation of cation transmembrane transport" (GO:1904062), and "Inorganic cation transmembrane transport" (GO:0098662). Previous studies have associated the alteration of pathways and functions related to cation transport with NAFLD [58]; however, information regarding relevance and significance remains scarce.

\section{Perspectives and significance}

Our results support the existence of differences between men and premenopausal women in specific biological processes and molecular functions relevant to NAFLD. Given the well-known heterogeneity of NAFLD progression between men and women, this transcriptomic meta-analysis suggests the use of sexspecific pathophysiologically informed disease biomarkers. These findings may also impact disease diagnosis and prognosis and the design of clinical trials. For example, biological sex-driven differences may allow the enhanced classification of individuals with NAFLD and alter their inclusion in specific clinical trials. Our meta-analysis underscores the importance of recognizing and accounting for sex-related differences in the study of NAFLD pathobiology.

\section{Conclusions}

While differences in clinical characteristics and pathogenic mechanisms in male and female NAFLD patients had been explored previously, details remained scarce. To this end, we selected seven studies suitable for functional meta-analysis and identified thirteen significant GO terms from these data. Of note, specific GO terms were previously identified to have relevance to NAFLD, which supports the notion that sex represents an important variable in understanding NAFLD pathobiology and heterogeneity. We identified novel functional terms that had not been linked to NAFLD previously; therefore, our study may open new avenues for further research. In conclusion, the meta-analysis of transcriptomic data represents a useful tool for the identification of sex-related differences in NAFL and NASH.

\section{Supplementary Information}

The online version contains supplementary material available at https://doi. org/10.1186/s13293-021-00368-1.

\footnotetext{
Additional file 1: Figure S1. UpSet plots of our gene functional analysis for each individual study for GO terms overexpressed in premenopausal women or in men and KEGG pathways upregulated in premenopausal women or in men.
}

Additional file 2: Figure S2. Forest plots for significant $\mathrm{GO}$ terms from our functional gene meta-analysis.

Additional file 3: Figure S3. Forest plots of significant GO terms from our functional pathways meta-analysis.

Additional file 4: Figure S4. Funnel plots of the significant GO terms from our functional gene meta-analysis.

Additional file 5: Figure S5. Funnel plots of significant $G O$ terms from our functional pathway meta-analysis.

Additional file 6: Supplementary table. Libraries version.

Additional file 7: Supplementary file. GEO Data.

\begin{abstract}
Abbreviations
BH: Benjamini \& Hochberg $p$ value adjusting method; GEO: Gene Expression Omnibus; GO: Gene Ontology; GSA: Gene Set Analysis; KEGG: Kyoto Encyclopedia of Genes and Genomes; LOR: Log odds ratio; NAFLD: Nonalcoholic fatty liver disease; NAFL: Non-alcoholic steatosis; NASH: Nonalcoholic steatohepatitis; PCA: Principal Component Analysis; PAA: Pathway Activity Analysis; PRISMA: Preferred Reporting Items for Systematic Reviews and Meta-Analyses; RMA: Robust Multichip Average
\end{abstract}

\section{Acknowledgements}

The authors would like to thank the Principe Felipe Research Centre (CIPF) for providing access to its computational cluster to carry out the work associated with this study. We would also like to thank Dr. Kristoffer T.G. Rigbolt for providing information regarding the ages of the samples used in study GSE126848.

\section{Authors' contributions}

FC analyzed the data; MRH and FGG designed and supervised the analysis; PMM designed and implemented the web tool; JFC, MRH, and FGG wrote the manuscript; MRH designed the graphical abstract; MB, GP, MIV, and HM helped in the interpretation of the results; MRH and FGG conceived the work. The authors read and approved the final manuscript.

\section{Funding}

This research was supported by grants from GV/2020/186 and ISCIII PT17/ 0009/0015 FEDER. Marina Berenguer was supported by a grant from the ISCIII (PI19/01360).

\section{Availability of data and materials}

The large volume of data and results generated in this study are freely available through the metafun-NAFLD web tool: https://bioinfo.cipf.es/ metafun-NAFLD. This study analyzed transcriptomic data available in the Gene Expression Omnibus database with accession numbers GSE48452, GSE61260, GSE66676, GSE83452, GSE89632, GSE126848, and GSE130970.

Ethics approval and consent to participate Not applicable

Consent for publication

Not applicable 


\section{Competing interests}

The authors declare no competing interests.

\section{Author details}

'Bioinformatics and Biostatistics Unit, Principe Felipe Research Center, Valencia, Spain. ${ }^{2}$ Spanish National Bioinformatics Institute, ELIXIR-Spain (INB, ELIXIR-ES), Madrid, Spain. ${ }^{3}$ Liver Transplantation and Hepatology Unit, Hospital Universitario y Politécnico La Fe, Valencia, Spain. ${ }^{4}$ Grupo de Hepatología, Cirugía HBP y Trasplantes, Instituto de Investigación Sanitaria La $\mathrm{Fe}$, Valencia, Spain. ${ }^{5} \mathrm{CIBER}$ hehd, Centro de Investigación Biomédica en Red en Enfermedades Hepáticas y Digestivas, Instituto de Salud Carlos III, Madrid, Spain. ${ }^{6}$ Department of Medicine, Universitat de València, Valencia, Spain. ${ }^{7}$ Division of Gastroenterology and Hepatology, Mayo Clinic, Rochester, MN, USA. ${ }^{8}$ Atos Research \& Innovation (ARI), Madrid, Spain. ${ }^{9}$ Biomedical Imaging Unit FISABIO-CIPF, Fundación para el Fomento de la Investigación Sanitario y Biomédica de la Comunidad Valenciana, Valencia, Spain.

Received: 25 November 2020 Accepted: 18 February 2021

Published online: 25 March 2021

\section{References}

1. Friedman SL, Neuschwander-Tetri BA, Rinella M, Sanyal AJ. Mechanisms of NAFLD development and therapeutic strategies. Nat Med. 2018;24:908-22. https://doi.org/10.1038/s41591-018-0104-9.

2. Younossi ZM, Koenig AB, Abdelatif D, Fazel Y, Henry L, Wymer M. Global epidemiology of nonalcoholic fatty liver disease-meta-analytic assessment of prevalence, incidence, and outcomes. Hepatology. 64:73-84. https://doi. org/10.1002/hep.28431.

3. Singh S, Allen AM, Wang Z, Prokop L, Murad MH, Loomba R. Fibrosis progression in nonalcoholic fatty liver vs nonalcoholic steatohepatitis: a systematic review and meta-analysis of paired-biopsy studies. Clin Gastroenterol Hepatol. 2015;13:643-54.e9. https://doi.org/10.1016/j.cgh.2014. 04.014.

4. Ballestri S, Nascimbeni F, Baldelli E, Marrazzo A, Romagnoli D, Lonardo A. NAFLD as a sexual dimorphic disease: role of gender and reproductive status in the development and progression of nonalcoholic fatty liver disease and inherent cardiovascular risk. Adv Ther. 2017;34:1291-326. https://doi.org/10.1007/s12325-017-0556-1.

5. Skubic C, Drakulić Ž, Rozman D. Personalized therapy when tackling nonalcoholic fatty liver disease: a focus on sex, genes, and drugs. Expert Opin Drug Metab Toxicol. 2018;14:831-41. https://doi.org/10.1080/174252 55.2018.1492552.

6. Zheng D, Wang X, Antonson P, Gustafsson J-Å, Li Z. Genomics of sex hormone receptor signaling in hepatic sexual dimorphism. Mol Cell Endocrinol. Elsevier. 2018;471:33-41. https://doi.org/10.1016/j.mce.2017.05.02 5.

7. Ling G, Sugathan A, Mazor T, Fraenkel E, Waxman DJ. Unbiased, genomewide in vivo mapping of transcriptional regulatory elements reveals sex differences in chromatin structure associated with sex-specific liver gene expression. Mol Cell Biol. 2010;30:5531-44. https://doi.org/10.1128/MCB. 00601-10.

8. Lonardo A, Suzuki A. Nonalcoholic fatty liver disease: Does sex matter? HepatoBiliary Surg Nutr. 2019;8:164-6. https://doi.org/10.21037/hbsn.201 8.12 .04

9. Barrett T, Wilhite SE, Ledoux P, Evangelista C, Kim IF, Tomashevsky M, et al. NCBI GEO: archive for functional genomics data sets-update. Nucleic Acids Res. 2012;41:D991-5. https://doi.org/10.1093/nar/gks1193.

10. Moher D, Liberati A, Tetzlaff J, Altman DG. Preferred Reporting Items for Systematic Reviews and Meta-Analyses: the PRISMA statement. PLoS Med. 2009;6:e1000097. https://doi.org/10.1371/journal.pmed.1000097.

11. Aller R, Fernández-Rodríguez C, lo lacono O, Bañares R, Abad J, Carrión JA, et al. Consensus document. Management of non-alcoholic fatty liver disease (NAFLD). Clinical practice guideline. Gastroenterol y Hepatol. 2018;41:32849. https://doi.org/10.1016/j.gastre.2018.05.013

12. R Core Team. R: A Language and environment for statistical computing. Vienna, Austria; 2019. http://www.r-project.org/

13. Bolstad BM, Irizarry RA, Astrand M, Speed TP. A comparison of normalization methods for high density oligonucleotide array data based on variance and bias. Bioinformatics. 2003;19:185-93. https://doi.org/10.1093/bioinformatics/1 9.2.185.
14. Robinson MD, Oshlack A. A scaling normalization method for differential expression analysis of RNA-seq data. Genome Biol. 2010;11:R25. https://doi. org/10.1186/gb-2010-11-3-r25.

15. Davis SR, Lambrinoudaki I, Lumsden M, Mishra GD, Pal L, Rees M, et al. Menopause. Nat Rev Dis Prim. 2015;1:15004. https://doi.org/10.1038/nrdp.2 015.4.

16. Freeman EW, Sammel MD, Sanders RJ. Risk of long-term hot flashes after natural menopause. Menopause. 2014;21:924-32. https://doi.org/10.1097/ GME.0000000000000196.

17. Ritchie ME, Phipson B, Wu D, Hu Y, Law CW, Shi W, et al. limma powers differential expression analyses for RNA-sequencing and microarray studies. Nucleic Acids Res. Oxford University Press. 2015;43:e47. https://doi.org/10.1 093/nar/gkv007.

18. Robinson MD, McCarthy DJ, Smyth GK. edgeR: a bioconductor package for differential expression analysis of digital gene expression data. Bioinformatics. 2010;26:139-40. https://doi.org/10.1093/bioinformatics/btp616.

19. Benjamini Y, Hochberg Y. Controlling the false discovery rate: a practical and powerful approach to multiple testing. J R Stat Soc Ser B. 1995;57:289-300.

20. Subramanian A, Tamayo P, Mootha VK, Mukherjee S, Ebert BL, Gillette MA, et al. Gene set enrichment analysis: a knowledge-based approach for interpreting genome-wide expression profiles. Proc Natl Acad Sci. 2005;102: 15545-50. https://doi.org/10.1073/pnas.0506580102

21. Montaner D, Dopazo J. Multidimensional gene set analysis of genomic data PLoS One. 2010;5:e10348. https://doi.org/10.1371/journal.pone.0010348.

22. Ashburner M, Ball CA, Blake JA, Botstein D, Butler H, Cherry JM, et al. Gene Ontology: tool for the unification of biology. Nat Genet. 2000;25:25-9. https://doi.org/10.1038/75556.

23. Kanehisa M, Goto S. KEGG: Kyoto Encyclopedia of Genes and Genomes. Nucleic Acids Res. 2000;28:27-30. https://doi.org/10.1093/nar/27.1.29.

24. Lex A, Gehlenborg N. Sets and intersections. Nat Methods. 2014;11:779. https://doi.org/10.1038/nmeth.3033.

25. Hidalgo MR, Cubuk C, Amadoz A, Salavert F, Carbonell-Caballero J, Dopazo $J$. High throughput estimation of functional cell activities reveals disease mechanisms and predicts relevant clinical outcomes. Oncotarget. 2017;8: 5160-78. https://doi.org/10.18632/oncotarget.14107

26. The UniProt Consortium. UniProt: the universal protein knowledgebase. Nucleic Acids Res. 2016;45:D158-69. https://doi.org/10.1093/nar/gkw1099.

27. García-García F. Métodos de análisis de enriquecimiento funcional en estudios genómicos: Universidad de Valencia; 2016. https://www.educacion. gob.es/teseo/mostrarRef.do?ref=1307283.

28. Viechtbauer W. Conducting meta-analyses in $\mathrm{R}$ with the metafor package. J Stat Softw]. 2010;36. https://doi.org/10.18637/jss.V036.103.

29. Schmidt FL, Hunter JE. Methods of meta-analysis: correcting error and bias in research findings. 3rd ed. SAGE Publications; 2015. https://doi.org/10.413 5/9781483398105

30. Ahrens M, Ammerpohl O, von Schönfels W, Kolarova J, Bens S, Itzel T, et al. DNA methylation analysis in nonalcoholic fatty liver disease suggests distinct disease-specific and remodeling signatures after bariatric surgery. Cell Metab. 2013;18:296-302. https://doi.org/10.1016/j. cmet.2013.07.004.

31. Horvath S, Erhart W, Brosch M, Ammerpohl O, von Schonfels W, Ahrens M, et al. Obesity accelerates epigenetic aging of human liver. Proc Natl Acad Sci. 2014;111:15538-43. https://doi.org/10.1073/pnas.1412759111.

32. Xanthakos SA, Jenkins TM, Kleiner DE, Boyce TW, Mourya R, Karns R, et al. High Prevalence of nonalcoholic fatty liver disease in adolescents undergoing bariatric surgery. Gastroenterology. 2015;149:623-34.e8. https:// doi.org/10.1053/j.gastro.2015.05.039

33. Lefebvre P, Lalloyer F, Baugé E, Pawlak M, Gheeraert C, Dehondt H, et al. Interspecies NASH disease activity whole-genome profiling identifies a fibrogenic role of PPARa-regulated dermatopontin. JCI Insight. Am Soc Clin Invest. 2017;2(13):e92264. https://doi.org/10.1172/jci.insight. 92264.

34. Arendt BM, Comelli EM, Ma DWL, Lou W, Teterina A, Kim T, et al. Altered hepatic gene expression in nonalcoholic fatty liver disease is associated with lower hepatic n-3 and n-6 polyunsaturated fatty acids. Hepatology. 2015;61:1565-78. https://doi.org/10.1002/hep.27695.

35. Suppli MP, Rigbolt KTG, Veidal SS, Heebøll S, Eriksen PL, Demant M, et al. Hepatic transcriptome signatures in patients with varying degrees of nonalcoholic fatty liver disease compared with healthy normal-weight individuals. Am J Physiol Gastrointest Liver Physiol. 2019;316:G462-72. https://doi.org/10.1152/ajpgi.00358.2018. 
36. Hoang SA, Oseini A, Feaver RE, Cole BK, Asgharpour A, Vincent R, et al. Gene expression predicts histological severity and reveals distinct molecular profiles of nonalcoholic fatty liver disease. Sci Rep. 2019;9:12541. https://doi. org/10.1038/s41598-019-48746-5.

37. Ryaboshapkina M, Hammar M. Human hepatic gene expression signature of non-alcoholic fatty liver disease progression, a meta-analysis. Sci Rep. 2017;7: 12361. https://doi.org/10.1038/s41598-017-10930-w.

38. Ruigrok ANV, Salimi-Khorshidi G, Lai M-C, Baron-Cohen S, Lombardo MV Tait RJ, et al. A meta-analysis of sex differences in human brain structure. Neurosci Biobehav Rev. 2014;39:34-50. https://doi.org/10.1016/j.neubiorev.2 013.12 .004$.

39. Casanova Ferrer F, Pascual M, Hidalgo MR, Malmierca-Merlo P, Guerri C, García-García F. Unveiling sex-based differences in the effects of alcohol aAbuse: a comprehensive functional meta-analysis of transcriptomic studies. Genes (Basel). 2020;11:1106. https://doi.org/10.3390/genes11091106.

40. Konishi S, Yano T, Tanaka H, Mizuno T, Kanoh H, Tsukita K, et al. Vinculin is critical for the robustness of the epithelial cell sheet paracellular barrier for ions. Life Sci Alliance. 2019;2:e201900414. https://doi.org/10.26508/lsa.201 900414.

41. Peng X, Nelson ES, Maiers JL, DeMali KA. Chapter five - new insights into vinculin function and regulation. In: Jeon KW, editor. Academic Press; 2011. p. 191-231. doi:https://doi.org/10.1016/B978-0-12-386043-9.00005-0.

42. Vandel J, Dubois-Chevalier J, Gheeraert C, Derudas B, Raverdy V, Thuillier D, et al. Hepatic molecular signatures highlight the sexual dimorphism of NonAlcoholic SteatoHepatitis (NASH). Hepatology. 2020;hep.31312. doi:https:// doi.org/10.1002/hep.31312.

43. Ward NC, Yu A, Moro A, Ban Y, Chen X, Hsiung S, et al. IL-2/CD25: a longacting fusion protein that promotes immune tolerance by selectively targeting the IL-2 receptor on regulatory T cells. J Immunol. 2018;201:257992. https://doi.org/10.4049/jimmunol.1800907.

44. Waters RS, Perry JSA, Han S, Bielekova B, Gedeon T. The effects of interleukin-2 on immune response regulation. Math Med Biol. 2018;35:79119. https://doi.org/10.1093/imammb/dqw021.

45. Saxena S, Nouri-Aria KT, Anderson MG, Eddleston AL, Williams R. Interleukin 2 activity in chronic liver disease and the effect of in vitro alpha-interferon. Clin Exp Immunol. 1986;63:541-8.

46. Perito ER, Ajmera V, Bass NM, Rosenthal P, Lavine JE, Schwimmer JB, et al. Association between cytokines and liver histology in children with nonalcoholic fatty liver disease. Hepatol Commun. 2017;1:609-22. https:// doi.org/10.1002/hep4.1068.

47. Wieckowska A, Papouchado BG, Li Z, Lopez R, Zein NN, Feldstein AE. Increased hepatic and circulating interleukin-6 levels in human nonalcoholic steatohepatitis. Am J Gastroenterol. 2008;103:1372-9.

48. Braunersreuther V, Viviani GL, Mach F, Montecucco F. Role of cytokines and chemokines in non-alcoholic fatty liver disease. World J Gastroenterol. 2012; 18:727. https://doi.org/10.3748/wjg.v18.i8.727.

49. Schmidt-Arras D, Rose-John S. IL-6 pathway in the liver: from physiopathology to therapy. J Hepatol. 2016;64:1403-15. https://doi.org/10.1 016/j.jhep.2016.02.004.

50. Yamaguchi K, Itoh Y, Yokomizo C, Nishimura T, Niimi T, Fujii H, et al. Blockade of interleukin-6 signaling enhances hepatic steatosis but improves liver injury in methionine choline-deficient diet-fed mice. Lab Investig. 2010; 90:1169-78. https://doi.org/10.1038/labinvest.2010.75.

51. Wang X, Yan Z, Ye Q. Interleukin-6 gene polymorphisms and susceptibility to liver diseases: a meta-analysis. Medicine (Baltimore). 2019;98:e18408. doi: https://doi.org/10.1097/MD.0000000000018408

52. Lonardo A, Nascimbeni F, Ballestri S, Fairweather D, Win S, Than TA, et al. Sex differences in nonalcoholic fatty liver disease: state of the art and identification of research gaps. Hepatology. 2019;70:1457-69. https://doi. org/10.1002/hep.30626.

53. Herrera-Marcos LV, Sancho-Knapik S, Gabás-Rivera C, Barranquero C, Gascón

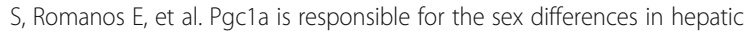
Cidec/Fsp27 $\beta$ mRNA expression in hepatic steatosis of mice fed a Western diet. Am J Physiol Metab. 2020;318:E249-61. https://doi.org/10.1152/a jpendo.00199.2019.

54. Matsusue K. A physiological role for fat specific protein 27/cell deathinducing DFF45-like effector C in adipose and liver. Biol Pharm Bull. 2010;33: 346-50. https://doi.org/10.1248/bpb.33.346.

55. Feldstein AE, Canbay A, Angulo P, Taniai M, Burgart LJ, Lindor KD, et al. Hepatocyte apoptosis and fas expression are prominent features of human nonalcoholic steatohepatitis. Gastroenterology. 2003;125:437-43. https://doi. org/10.1016/s0016-5085(03)00907-7.

56. Russell JO, Monga SP. Wnt/ $\beta$-catenin signaling in liver development, homeostasis, and pathobiology. Annu Rev Pathol. 2018;13:351-78. https:// doi.org/10.1146/annurev-pathol-020117-044010.

57. Zhu L, Baker SS, Shahein A, Choudhury S, Liu W, Bhatia T, et al. Upregulation of non-canonical Wnt ligands and oxidative glucose metabolism in NASH induced by methionine-choline deficient diet. Trends cell Mol Biol. 2018;13: 47-56. https://doi.org/10.31300/tcmb.13.2018.47-56.

58. Pirola CJ, Sookoian S. Multiomics biomarkers for the prediction of nonalcoholic fatty liver disease severity. World J Gastroenterol. 2018;24: 1601-15. https://doi.org/10.3748/wjg.v24.i15.1601.

\section{Publisher's Note}

Springer Nature remains neutral with regard to jurisdictional claims in published maps and institutional affiliations.
Ready to submit your research? Choose BMC and benefit from:

- fast, convenient online submission

- thorough peer review by experienced researchers in your field

- rapid publication on acceptance

- support for research data, including large and complex data types

- gold Open Access which fosters wider collaboration and increased citations

- maximum visibility for your research: over $100 \mathrm{M}$ website views per year

At BMC, research is always in progress.

Learn more biomedcentral.com/submissions 